\title{
In-Situ TEM Observation of Metal Zn Nanocrystal Growth on ZnO Films
}

Jong Min Yuk*, Kwanpyo Kim*****, Zonghoon Lee****, Masashi Watanabe*****, Alex Zettl*****, Jeong Yong Lee*, Young Soo No******, Tae Whan Kim******, and Won Kook Choi $* * * * * * *$

* Dept. of Mater. Sci. \& Eng., Korea Advanced Institute of Science and Technology, Daejeon 305701, Korea

** Dept. of Phys./Center of Integrated Nanomechanical Systems, UC Berkeley, CA94720, USA

*** Materials Sciences Division, **** National Center for Electron Microscopy, Lawrence Berkeley National Laboratory, Berkeley, CA94720, USA

***** Dept. of Mater. Sci. \& Eng., Lehigh University, Bethlehem, PA18015, USA

****** National Research Laboratory for Nano Quantum Electronics Laboratory, Division of Electronics \& Computer Eng., Hanyang University, Seoul 133-791, Korea

******* Thin Film Materials Research Center, Korea Institute of Science and Technology, Seoul 136-791, Korea

The nanocrystal formation has attracted significant attention due to their unique properties and their potential applications to electronic and optoelectronic devices operating with lower currents at higher temperatures [1]. Extensive efforts have been made to fabricate various kinds of nanocrystals on semiconductor substrates. Recently, proximal probes in scanning tunneling microscopy (STM) and atomic force microscopy (AFM) have been suggested as potential tools for the nanocrystal fabrications together with their capabilities of atomic-level imaging and manipulating [2]. One of the most promising techniques is a local anodic-oxidation method using AFM/STM, which can produce a pattern of nanocrystal arrays on surface of semiconductor substrate for fabricating nano-devices [3]. However, the local anodic-oxidation mechanism with elemental rearrangements has not yet been established. Therefore, it is important to investigate the anodic-oxidation mechanism for nanocrystal formation. In this study, the formations of $\mathrm{ZnO}$ and $\mathrm{Zn}$ nanocrystals on $\mathrm{ZnO}$ film through currentAFM (C-AFM) and biasing tungsten-tip were characterized both in ex- and in-situ transmission electron microscopy (TEM). In-situ TEM was performed with a biasing tungsten-tip specimen holder. This in-situ approach allows us not only to observe nanocrystal growth in live but also to characterize microstructure rearrangements by electron diffraction followed by elemental analysis.

Figure 1 shows a set of AFM images of (a) as-grown $\mathrm{ZnO}$ film on a $n$-Si (001) substrate, $\mathrm{ZnO}$ films after applying C-AFM (b) line- and (c) dot-oxidation on the as-grown $\mathrm{ZnO}$ film. In addition to the AFM images, a cross-sectional TEM image of the $\mathrm{ZnO}$ film after applying C-AFM dot-oxidation is also shown in Fig. 1(d). When a C-AFM conductive tip with a sample voltage of $10 \mathrm{~V}$ is positioned on the $\mathrm{ZnO}$ film surface, additional oxide layer is formed at the location because $\mathrm{OH}^{-}$and $\mathrm{O}^{2-}$ ions may be adsorbed onto the surface due to a strong electric field between the tip and the sample [4]. Furthermore, $\mathrm{Zn}$ nanocrystals can be fabricated using biasing tungsten-tip in in-situ TEM. In this approach, the size, position and shape of Zn nanocrystals can be controlled. A schematic diagram of the nanocrystal formation by biasing tip is shown in Fig. 2(a). A bright-field TEM image after applying bias to the $\mathrm{ZnO}$ film in in-situ TEM indicates the $\mathrm{Zn}$ nanocrystal formation. Further nanocrystal formation mechanisms based on in-situ TEM observation will be discussed [5]. 


\section{References}

[1] N. J. Craig et al., Science 304 (2004) 565.

[2] D. M. Eigler et al., Nature (London) 344 (1990) 524.

[3] R. H. Kim et al., Appl. Phys. Lett. 90 (2007) 172907.

[4] Y.-R. Ma et al., Phys. Rev. B 64 (2001) 195324.

[5] This work was supported by the National Center for Electron Microscopy, Lawrence Berkeley Lab, which is supported by the U.S. Department of Energy under Contract \# DE-AC02-05CH11231.
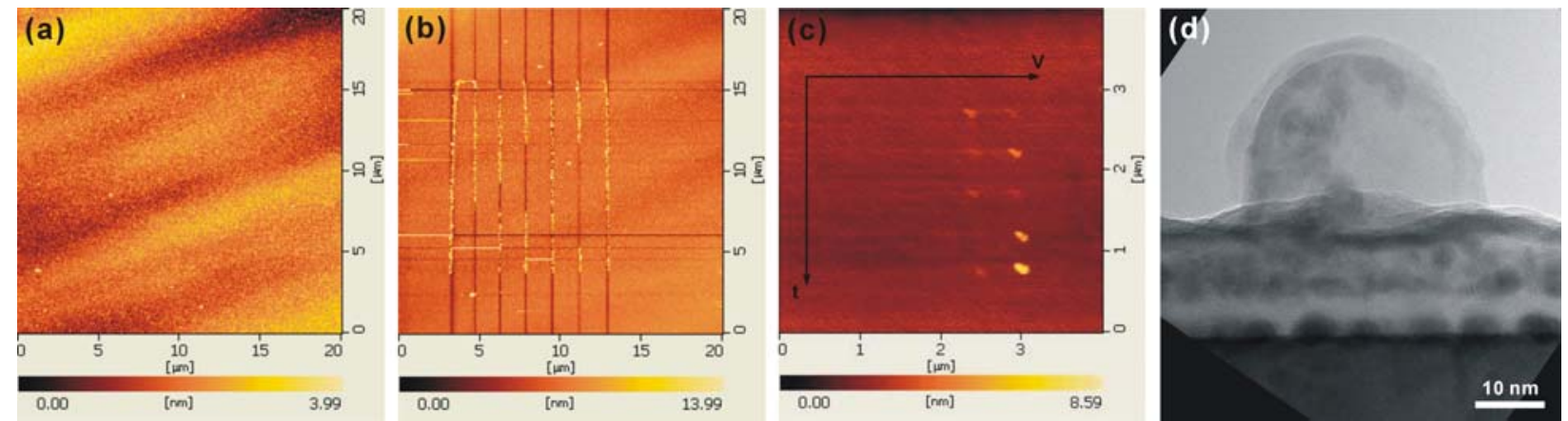

Fig. 1. (a) An AFM image of as-grown $\mathrm{ZnO} / n$-Si (001) heterostructures. AFM images of the $\mathrm{ZnO}$ film after C-AFM (b) line- and (c) dot-oxidation. (d) A cross-sectional TEM image of dot-oxidation in (c).
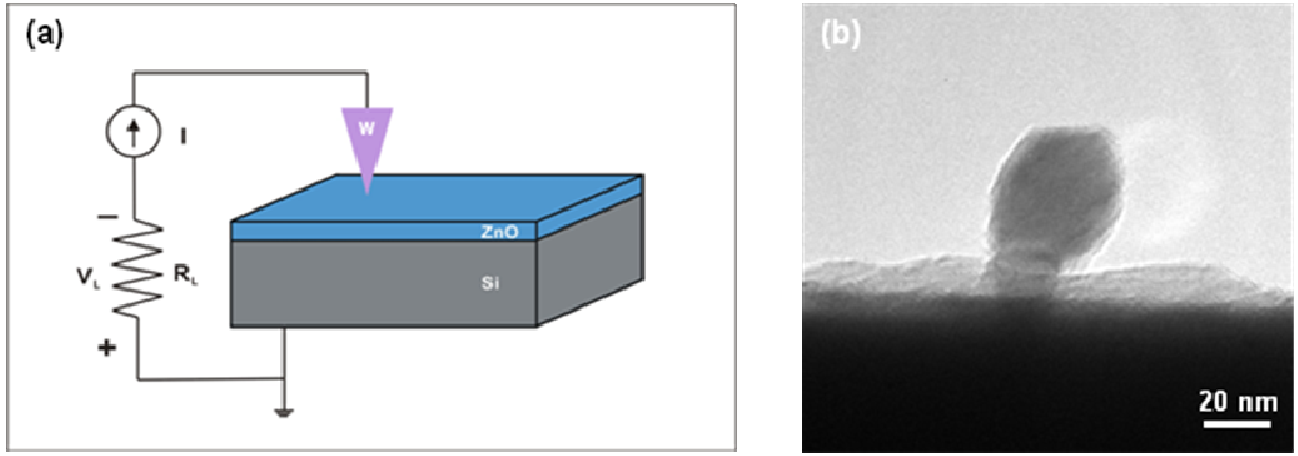

Fig. 2. (a) A schematic diagram of the fabrication $\mathrm{Zn}$ nanocrystals using a biasing tungsten-tip. (b) A bright-field TEM image of a Zn nanocrystal fabricated by in-situ TEM with the biasing tungsten-tip. 